\title{
Identification of the radicals formed in the reactions of some endogenous photosensitizers with oleic acid under the UVA irradiation
}

\author{
Hiroko Mori ${ }^{1,2}$ and Hideo Iwahashi ${ }^{1, *}$ \\ 'Department of Chemistry, Wakayama Medical University, 580 Mikazura, Wakayama 641-0011, Japan \\ ${ }^{2}$ Morinomiya College of Medical Arts and Sciences, 4-1-8 Nakamoto, Osaka 537-0022, Japan
}

(Received 8 December, 2011; Accepted 9 February, 2012; Published online 11 July, 2012)

\begin{abstract}
Electron spin resonance measurements were performed for the reactions of some endogenous photosensitizers (flavin mononucleotide or flavin adenine dinucleotide or folic acid or $\beta$-nicotinamide adenine dinucleotide or $\beta$-nicotinamide adenine dinucleotide phosphate or pyridoxal-5'-phosphate or urocanic acid) with oleic acid under the ultraviolet light $A$ irradiation using $\alpha$-(4-pyridyl-1oxide)- $N$-tert-butylnitrone as a spin trap reagent. Of the endogenous photosensitizers, prominent electron spin resonance signals $\left(\alpha^{\mathrm{N}}=1.58 \mathrm{mT}\right.$ and $\left.\alpha^{\mathrm{H}} \beta=0.26 \mathrm{mT}\right)$ were observed for the reaction mixture of flavin mononucleotide (or flavin adenine dinucleotide or folic acid), suggesting that radical species form in the reaction mixtures. Singlet oxygen seems to participate in the formation of the radicals because the electron spin resonance peak heights increased for the reactions in $\mathrm{D}_{2} \mathrm{O}$ to a great extent. A high performance liquid chromatography-electron spin resonance-mass spectrometry was employed to identify the radicals formed in the reactions of the endogenous photosensitizers (flavin mononucleotide or flavin adenine dinucleotide or folic acid) with oleic acid under the ultraviolet light $A$ irradiation. The high performance liquid chromatography-electron spin resonance-mass spectrometry analyses showed that 7-carboxyheptyl and 1-(3-carboxypropyl)-4hydroxybutyl radicals form in the reaction mixture of flavin mononucleotide (or flavin adenine dinucleotide or folic acid).
\end{abstract}

Key Words: singlet oxygen, lipid peroxidation, 7-carboxyheptyl radical, HPLC-ESR-MS, UVA

$\mathrm{O}$ $\mathrm{f}$ the various oxidative stresses, ultraviolet (UV) irradiation is one of the primary factors. The ultraviolet light A (UVA) component $(320-400 \mathrm{~nm})$ of solar radiation has been shown to produce deleterious biological effects in which singlet oxygen ${ }^{1} \mathrm{O}_{2}$ $\left({ }^{1} \Delta_{\mathrm{g}}\right)$ plays a major role. Yasui and Sakurai ${ }^{(1,2)}$ confirmed the generation of ${ }^{1} \mathrm{O}_{2}\left({ }^{1} \Delta \mathrm{g}\right)$ in the skin of live rodents exposed to UVA using chemiluminescent detection and imaging. This must have an effect on all tissue that gets into contact with UVA, particularly the skin and the eye.

There seems to be a mechanism for the generation of ${ }^{1} \mathrm{O}_{2}\left({ }^{1} \Delta_{\mathrm{g}}\right)$ in biological systems. The type-II reaction of a photosensitizer transfers its energy to a ground state oxygen molecule to form ${ }^{1} \mathrm{O}_{2}$ $\left({ }^{1} \Delta_{\mathrm{g}}\right) .^{(3)}$ Ragas et al. ${ }^{(4)}$ showed that singlet oxygen is produced in gram-negative Escherichia coli bacteria in the presence of a cationic porphyrin by a combination of spectroscopic and timeresolved photophysical techniques. On the other hand, singlet oxygen was directly detected in the solution of endogenous photosensitizers such as riboflavin, flavin mononucleotide (FMN), flavin adenine dinucleotide (FAD), $\beta$-nicotinamide adenine dinucleotide (NAD), $\beta$-nicotinamide adenine dinucleotide phosphate (NADP), pyridoxal-5'-phosphate (PLP), urocanic acid and cholesterol. ${ }^{(5-8)}$

Yamazaki et al. ${ }^{(9)}$ found that the product of the ${ }^{1} \mathrm{O}_{2}\left({ }^{1} \Delta \mathrm{g}\right)$-specific oxygenation of cholesterol, 3 $\beta$-hydroxy-5 $\alpha$-cholest-6-ene-5hydroperoxide, accumulated in the skin of rats treated with an oral dose of a photosensitizer, pheophorbide and subsequently exposed to visible light. The UV-induced lipid peroxidation also occurs through a non-radical pathway by a direct reaction with singlet oxygen $\left({ }^{1} \Delta_{\mathrm{g}}\right)^{(10-13)}$ in addition to a free radical chain reaction. ${ }^{(10,14,15)}$

Increasing electron spin resonance (ESR) evidences showed that free radicals may be involved in the acute sunburn reactions. Indeed, reactive oxygen speceis (ROS) such as hydroxyl radicals $\left(\mathrm{HO}^{*}\right)^{(16)}$ and superoxide anion radical $\left(\mathrm{O}_{2}{ }^{-*}\right)^{(17)}$ were detected under the UV irradiation using ESR spin trapping technique. Furthermore, 5,5-dimethyl-1-pyrroline- $N$-oxide (DMPO) or $\alpha$-(4pyridyl-1-oxide)- $N$-tert-butyl nitrone (4-POBN)/lipid-derived radical adducts were also observed in the epidermal homogenate of albino rat skins following UV exposure, ${ }^{(18,19)}$ in UV light exposed mouse skin, ${ }^{(20)}$ and in human skin exposed to solarirradiation. ${ }^{(21,22)}$

In spite of the intensive ESR studies, chemical structures of the radical species formed in the epidermal homogenate of albino rat skins following UV exposure, ${ }^{(18,19)}$ in UV light exposed mouse skin, ${ }^{(20)}$ and in human skin exposed to solar-irradiation have not been determined. ${ }^{(21,22)}$ In this study, identification of the lipidderived radicals is performed for the reactions of some endogenous photosensitizers with oleic acid under the UVA irradiation (365 nm) using ESR, HPLC-ESR and HPLC-ESR-MS. ${ }^{(23)}$ A spin trap reagent, 4-POBN was used to trap the lipid-derived radicals.

\section{Materials and Methods}

Materials. 4-POBN, FMN, urocanic acid, NAD, NADP and oleic acid were purchased from Tokyo Kasei Kogyo, Ltd. (Tokyo, Japan). FAD, cholic acid, deuterium oxide $\left(\mathrm{D}_{2} \mathrm{O}\right)$ and thymidine5'-monophospate (TMP) were purchased from Wako Pure Chem. Ind. Ltd. (Osaka, Japan). 2'-Deoxyguanosine-5'-monophospate (GMP), 2'-deoxycytidine-5'-monophospate (CMP), 4-oxo-2,2,6,6tetramethylpiperidine (4-oxo-TEMP) and PLP were from SigmaAldrich Co. (St. Louis, MO). 2'-Deoxyadenosine-5'-monophospate (AMP) was from Oriental Yeast Co. Ltd. (Tokyo, Japan). Water used in these experiments was purified by passing through Milli-Q

*To whom correspondence should be addressed. E-mail: chem1@wakayama-med.ac.jp 
Jr. (Nihon Millipore Kogyo K.K., Yonezawa, Japan) after distillation. All other chemicals used were of analytical grade.

ESR, HPLC-ESR, and HPLC-ESR-MS analyses. The ESR, HPLC-ESR, and HPLC-ESR-MS analyses were performed as described in our previous paper. ${ }^{(24)}$

Standard reaction mixture. The standard reaction mixture contained $4.3 \mathrm{mM}$ oleic acid, $25 \mu \mathrm{M}$ FMN (or FAD or folic acid or urocanic acid or NAD or NADP or PLP), $40 \mathrm{mM}$ phosphate buffer (pH 7.4), $10 \mathrm{mM}$ cholic acid, $0.1 \mathrm{M} 4-\mathrm{POBN}$ and $1 \mathrm{mM} \mathrm{FeSO}_{4}$ in a quartz test tube $(100 \mathrm{~mm}$ long $\times 8 \mathrm{~mm}$ i.d. $)$. The standard reaction mixtures without $0.1 \mathrm{M} 4-\mathrm{POBN}$ and $1 \mathrm{mM} \mathrm{FeSO}_{4}$ were exposed to the $7.8 \mathrm{~J} / \mathrm{cm}^{2}$ light of $365 \mathrm{~nm}$ using a REX 250 irradiation system with a LXO365 bandpass filter (ASAHI SPECTRA Co., Tokyo, Japan) unless otherwise noted. After the irradiation, $0.1 \mathrm{M} 4-\mathrm{POBN}$ was added. The reactions were started by adding $1 \mathrm{mM} \mathrm{FeSO}$. The reactions were performed at $25^{\circ} \mathrm{C}$ for $1 \mathrm{~min}$. The reaction mixtures were aspirated into a Teflon tube centered in an ESR microwave cavity. And then, ESR spectra were measured.

Reaction in $\mathrm{D}_{2} \mathrm{O}$. Reactions in $\mathrm{D}_{2} \mathrm{O}$ were performed as described in our previous paper. ${ }^{(24)}$

Ultraviolet and visible absorption spectra. Ultraviolet and visible absorption spectra were measured using a model UV-160A ultraviolet-visible spectrometer (Shimadzu Co., Kyoto, Japan). The spectrometer was operated from 300 to $550 \mathrm{~nm}$. The measurements were performed at $25^{\circ} \mathrm{C}$. The measurements were carried out in a cuvette with a $10 \mathrm{~mm}$ light path. In the reference cell, $200 \mathrm{mM}$ phosphate buffer ( $\mathrm{pH}$ 7.4) was contained. The ultraviolet and visible measurements were performed for the mixtures of $50 \mu \mathrm{M}$ FMN in the $200 \mathrm{mM}$ phosphate buffer.

Fluorescence spectroscopy. The fluorescence spectra of the samples were taken using a 650-10S fluorescence spectrophotometer (Hitachi, Ltd., Tokyo, Japan). The excitation wavelength used were $365 \mathrm{~nm}$. The range of emission wavelength is 450 to $700 \mathrm{~nm}$.

\section{Results}

ESR measurements of the standard reaction mixtures of some endogenous photosensitizers with oleic acid under the UVA irradiation. After $7.8 \mathrm{~J} / \mathrm{cm}^{2}$ (or $23.4 \mathrm{~J} / \mathrm{cm}^{2}$ ) irradiation of $365 \mathrm{~nm}$, ESR spectra of the standard reaction mixtures of some endogenous photosensitizers with oleic acid were measured (Fig. 1). Prominent ESR signals $\left(\alpha^{\mathrm{N}}=1.58 \mathrm{mT}\right.$ and $\left.\alpha^{\mathrm{H}} \beta=0.26 \mathrm{mT}\right)$ were observed for the standard reaction mixture of FMN (or FAD or folic acid) (Fig. $1 \mathrm{~A}-\mathrm{D}$ ), suggesting that free radicals form in the standard reaction mixture of FMN (or FAD or folic acid). The relative ESR peak height of FAD is $32 \pm 9 \%$ of the standard reaction mixture of FMN (Table 1). The relative ESR peak heights of folic acid are $38 \pm 7 \%\left(7.8 \mathrm{~J} / \mathrm{cm}^{2}\right)$ and $61 \pm 13 \%\left(23.4 \mathrm{~J} / \mathrm{cm}^{2}\right)$ of the standard reaction mixture of FMN, respectively (Table 1). The
ESR signals were hardly observed for the standard reaction mixture of FMN without the UVA $\left(365 \mathrm{~nm}\right.$ ) irradiation (or $\mathrm{Fe}^{2+}$ or FMN or oleic acid) (Fig. 2 B-E). This is also the case for the reaction mixture of FAD (or folic acid) (data not shown). To know whether singlet oxygens are involved in the radical formation, the reactions were performed in $\mathrm{D}_{2} \mathrm{O}$. The ESR peak heights of the reaction of FMN $\left(7.8 \mathrm{~J} / \mathrm{cm}^{2}\right)$ [or FAD $\left(7.8 \mathrm{~J} / \mathrm{cm}^{2}\right)$ or folic acid $\left.\left(23.4 \mathrm{~J} / \mathrm{cm}^{2}\right)\right]$ in $\mathrm{D}_{2} \mathrm{O}$ increased compared with the reactions in $\mathrm{H}_{2} \mathrm{O}$ (Table 1). Thus, singlet oxygen seems to be involved in the formation of the radicals because $\mathrm{D}_{2} \mathrm{O}$ increases the lifetime of singlet oxygen. ${ }^{(3)}$

The ESR peak heights under the UVA irradiations of the various wavelengths. After $7.8 \mathrm{~J} / \mathrm{cm}^{2}$ irradiations of the various wavelengths $(313,334,365,405$, and $436 \mathrm{~nm})$, the ESR spectra were measured for the standard reaction mixtures of FMN (Fig. 3). The relative ESR peak heights were as follows, $46 \pm 4 \%$ $(313 \mathrm{~nm}), 74 \pm 14 \%(334 \mathrm{~nm}), 88 \pm 8 \%(405 \mathrm{~nm})$ and $94 \pm 17 \%$

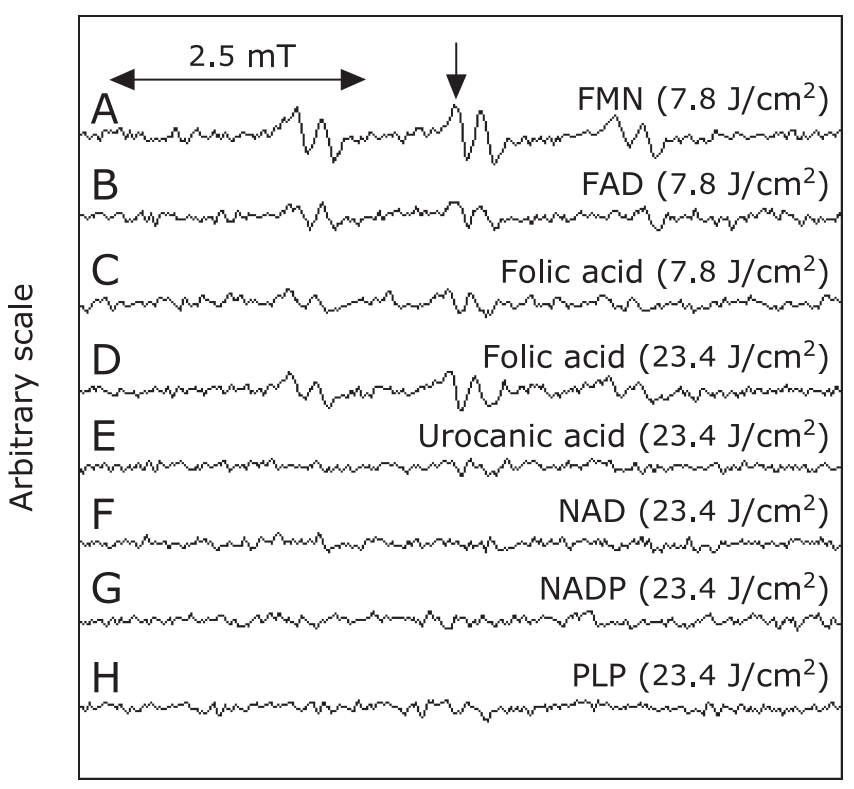

Fig. 1. ESR spectra of the standard reaction mixtures of some endogenous photosensitizers under the UVA irradiation. The standard reaction mixtures of FMN under UVA irradiation $\left(7.8 \mathrm{~J} / \mathrm{cm}^{2}\right)(A), F A D$ under UVA irradiation $\left(7.8 \mathrm{~J} / \mathrm{cm}^{2}\right)(B)$, folic acid under UVA irradiation $\left(7.8 \mathrm{~J} / \mathrm{cm}^{2}\right)(C)$, folic acid under UVA irradiation $\left(23.4 \mathrm{~J} / \mathrm{cm}^{2}\right)(D)$, urocanic acid under UVA irradiation $\left(23.4 \mathrm{~J} / \mathrm{cm}^{2}\right)(E)$, NAD under UVA irradiation $\left(23.4 \mathrm{~J} / \mathrm{cm}^{2}\right)(F)$, NADP under UVA irradiation $\left(23.4 \mathrm{~J} / \mathrm{cm}^{2}\right)(\mathrm{G})$, and PLP under UVA irradiation $\left(23.4 \mathrm{~J} / \mathrm{cm}^{2}\right)(\mathrm{H})$.

Table 1. Relative ESR peak heights of various endogeneous photosensitizers

\begin{tabular}{|c|c|c|}
\hline Photosensitizers ${ }^{(a)}$ & $\begin{array}{c}\text { Relative ESR peak } \\
\text { height }(\%) \pm \mathrm{SD}\left(\text { in } \mathrm{H}_{2} \mathrm{O}\right)^{(\mathrm{b})}\end{array}$ & $\begin{array}{c}\text { Relative ESR peak } \\
\text { height }(\%) \pm S D\left(\text { in } \mathrm{D}_{2} \mathrm{O}\right)^{(\mathrm{c})}\end{array}$ \\
\hline FMN $\left(7.8 \mathrm{~J} / \mathrm{cm}^{2}\right)$ & 100 & $336 \pm 78$ \\
\hline FAD $\left(7.8 \mathrm{~J} / \mathrm{cm}^{2}\right)$ & $32 \pm 9$ & $89 \pm 10$ \\
\hline Folic acid $\left(7.8 \mathrm{~J} / \mathrm{cm}^{2}\right)$ & $38 \pm 7$ & - \\
\hline Folic acid $\left(23.4 \mathrm{~J} / \mathrm{cm}^{2}\right)$ & $61 \pm 13$ & $260 \pm 33$ \\
\hline Urocanic acid $\left(23.4 \mathrm{~J} / \mathrm{cm}^{2}\right)$ & 0 & 0 \\
\hline NAD $\left(23.4 \mathrm{~J} / \mathrm{cm}^{2}\right)$ & 0 & 0 \\
\hline $\operatorname{NADP}\left(23.4 \mathrm{~J} / \mathrm{cm}^{2}\right)$ & 0 & 0 \\
\hline PLP $\left(23.4 \mathrm{~J} / \mathrm{cm}^{2}\right)$ & 0 & 0 \\
\hline
\end{tabular}

The data presented are the percentages in comparison with the ESR peak height of the standard reaction mixture of FMN. The data are mean \pm SD of three independent experiments. Irradiation energies are shown in the parentheses. ${ }^{(a)}$ The reactions were performed in $\mathrm{H}_{2} \mathrm{O}^{(\mathrm{b})}$ or $\mathrm{D}_{2} \mathrm{O}$.(c) 
$(436 \mathrm{~nm})$ of the ESR peak height $(365 \mathrm{~nm})$. Since absorbances of FMN at the respective wavelengths are $0.103(313 \mathrm{~nm}), 0.246$ $(334 \mathrm{~nm}), 0.496(365 \mathrm{~nm}), 0.361(405 \mathrm{~nm})$ and $0.588(436 \mathrm{~nm})$, the reaction is closely related to the ultraviolet-visible absorbance of FMN (Fig. 4). The FMN seems to work in the reactions as a photosensitizer.

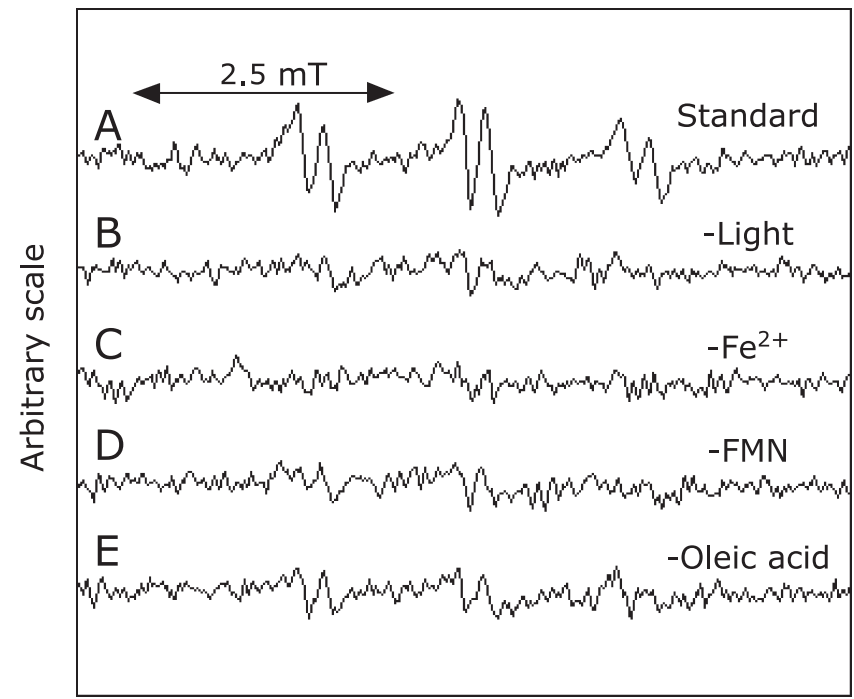

Fig. 2. ESR spectra of the standard reaction mixtures of FMN under the UVA irradiation. The standard reaction mixtures of FMN (A), FMN without light (B), FMN without $\mathrm{Fe}^{2+}(\mathrm{C}), \mathrm{FMN}$ without $\mathrm{FMN}(\mathrm{D})$, and FMN without oleic acid (E).

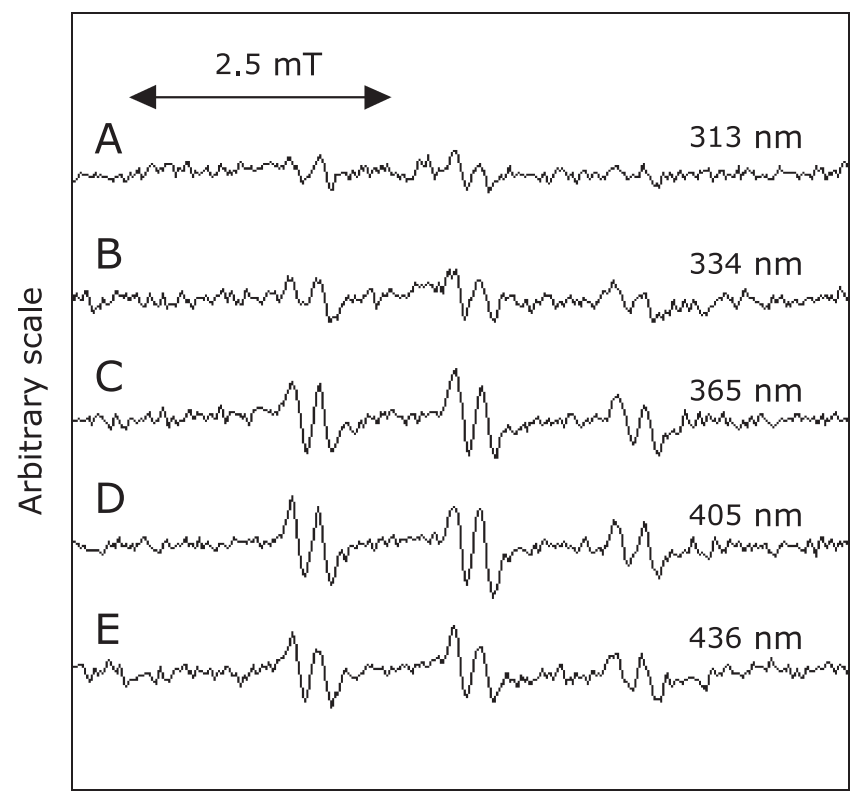

Fig. 3. The ESR spectra under the UVA irradiation of various wavelengths. The standard reaction mixtures of FMN without both $0.1 \mathrm{M}$ 4-POBN and $1 \mathrm{mM} \mathrm{FeSO}_{4}$ were exposed to the various wavelength lights $\left(7.8 \mathrm{~J} / \mathrm{cm}^{2}\right)$ using a REX 250 irradiation system with a LXO $313(313 \mathrm{~nm})$ [or LXO334 $(334 \mathrm{~nm})$ or LXO365 $(365 \mathrm{~nm})$ or LHO405 $(405 \mathrm{~nm})$ or LHO436 $(436 \mathrm{~nm})$ ] bandpass filter (ASAHI SPECTRA Co., Tokyo, Japan). (A) $313 \mathrm{~nm}$. (B) $334 \mathrm{~nm}$. (C) $365 \mathrm{~nm}$. (D) $405 \mathrm{~nm}$. (E) $436 \mathrm{~nm}$.
Irradiation energy dependence of the ESR peak heights. Irradiation energy dependence of the ESR peak height was measured for the standard reaction mixtures of FMN (or FAD) (Fig. 5). The ESR peak heights increased with the increase of the irradiation energy for the standard reaction mixtures of FMN, and reached $2.8 \mathrm{~cm}$ at $15.6 \mathrm{~J} / \mathrm{cm}^{2}$. On the other hand, the ESR peak height increased with increase of the irradiation energy for the standard reaction mixtures of $F A D$, and reached a plateau $(1.0 \mathrm{~cm})$ at $1.95 \mathrm{~J} / \mathrm{cm}^{2}$, suggesting that FAD is a weak phtosensitizer compared with FMN.

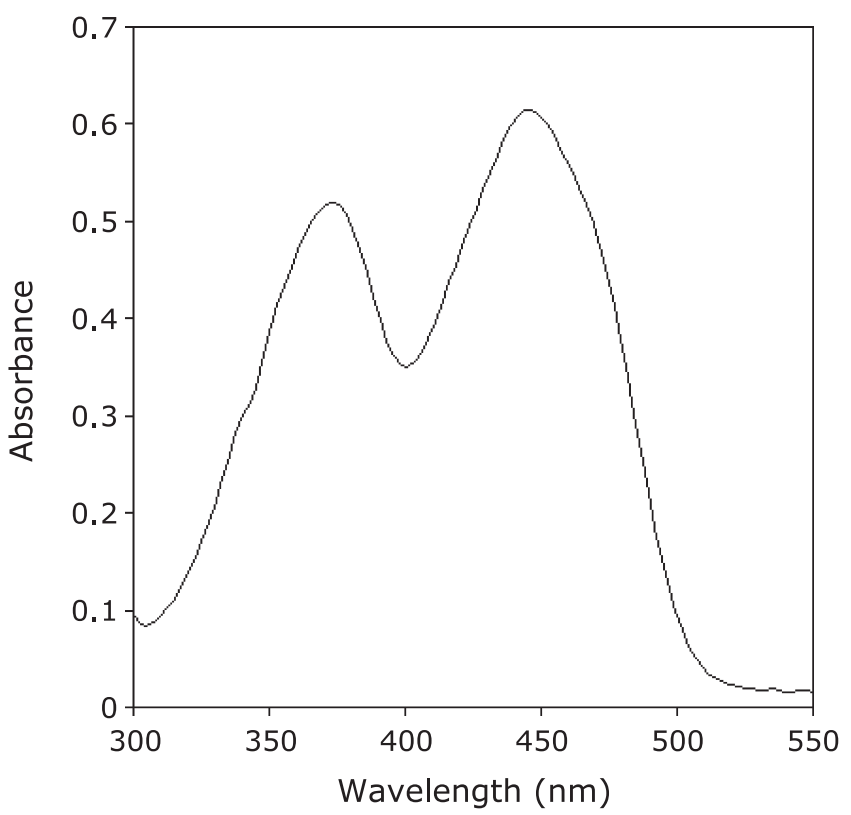

Fig. 4. An ultraviolet and visible absorption spectrum of FMN.

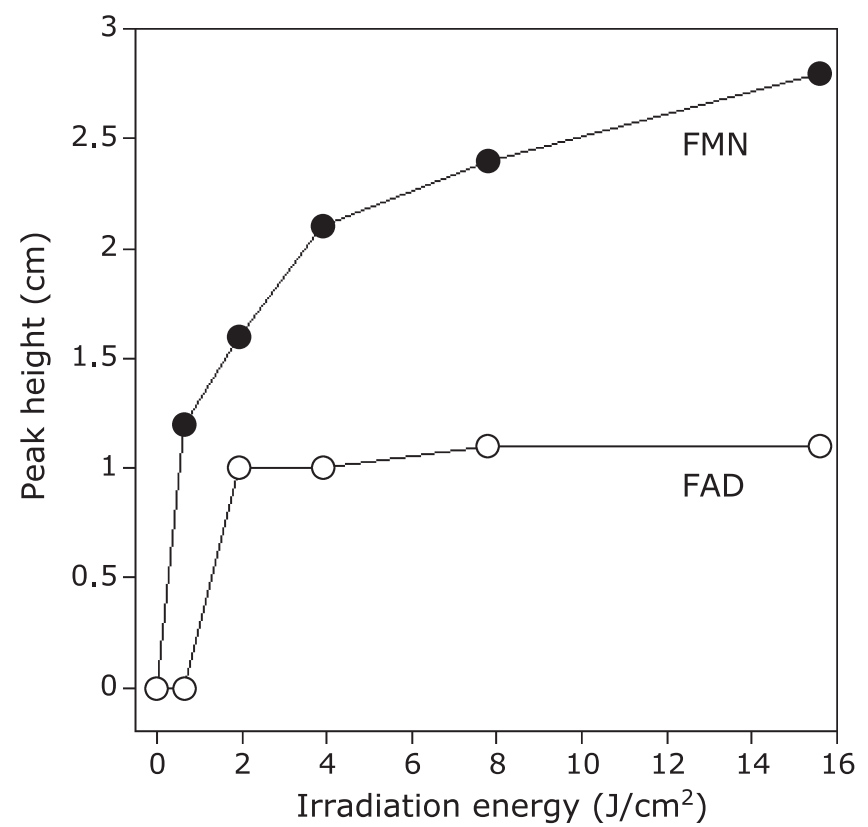

Fig. 5. Irradiation energy dependence of the ESR peak heights. Standard reaction mixture of FMN (-). Standard reaction mixture of FAD $(-\circ)$. 
Measurments of fluorescence spectra of FMN and FAD. To know whether or not adenosine moiety in the FAD quenches the singlet excited states of FAD, fluorescence spectra were measured for the standard reaction mixture of FMN (or FAD) without both ferrous ions and 4-POBN (Fig. 6). An intense fluorescence spectrum was observed for the solution of FMN. While, FAD showed a weak fluorescence spectrum. In order to clarify the effect of adenosine moiety in FAD on the fluorescence spectrum, fluorescence spectra were measured by adding various concentrations of AMP to the standard reaction mixture of FMN without both ferrous ions and 4-POBN (Fig. 7). The intensity of fluorescence spectra decreased with the increase of the concentration of AMP, suggesting that AMP quenches the fluorescence of FMN. At $26.7 \mathrm{mM}$ of AMP, the intensity of the fluorescence decreased to $46.6 \pm 1.6 \%$ of the standard reaction mixture without both ferrous ions and 4-POBN.

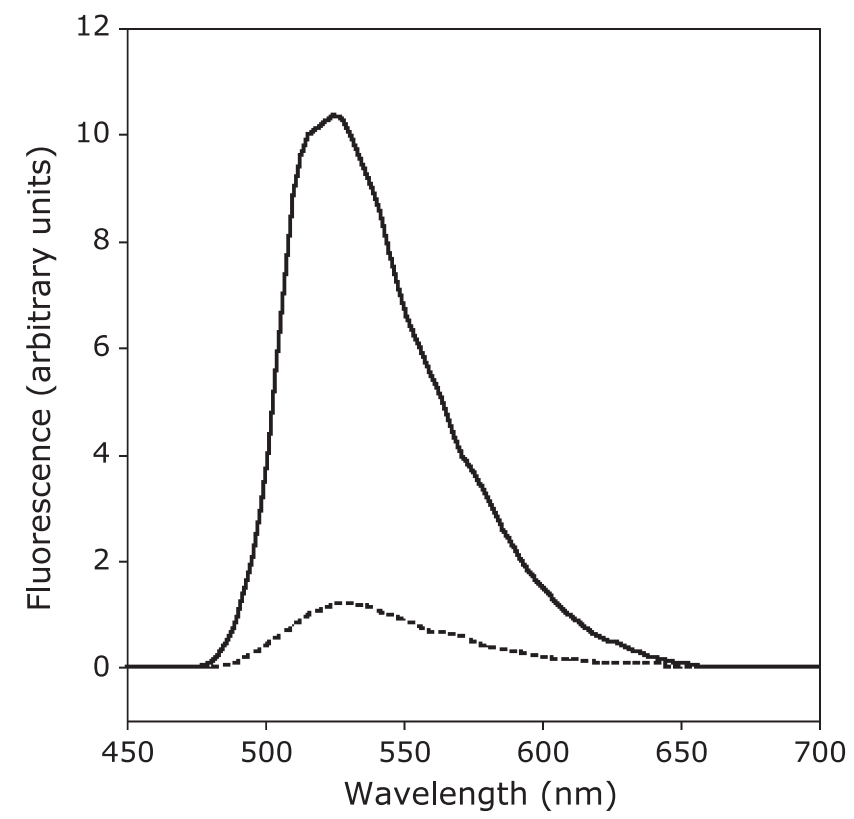

Fig. 6. Fluorescence spectra of FMN and FAD. FMN and FAD were dissolved in $0.2 \mathrm{M}$ phosphate buffer ( $\mathrm{pH}$ 7.4). $5 \mu \mathrm{M}$ FMN (-). $5 \mu \mathrm{M}$ FAD (---).
The effect of AMP on the formation of 4-POBN radical adduct and 4-oxo-2,2,6,6-tetramethylpiperidine- $N$-oxyl (4oxo-TEMPO). Since AMP quenches the fluorescence of FMN, effects of AMP on the formation of the radicals and singlet oxygen ${ }^{1} \mathrm{O}_{2}$ were examined. We used the trap probe 4-oxo-TEMP to detect ${ }^{1} \mathrm{O}_{2}$. This probe, which has been shown to be specific for ${ }^{1} \mathrm{O}_{2}$ detection, ${ }^{(25,26)}$ reacts with ${ }^{1} \mathrm{O}_{2}$ to yield a stable nitroxide radical 4oxo-2,2,6,6-tetramethylpiperidine- $N$-oxyl (4-oxo-TEMPO), having a known three-line ESR spectrum. The stable nitroxide radical 4-oxo-TEMPO was detected in the standard reaction mixture of FMN without $\mathrm{Fe}^{2+}$ and 4-POBN with $0.1 \mathrm{M}$ 4-oxo-TEMP. On addition of AMP to the standard reaction mixture without $\mathrm{Fe}^{2+}$ and 4-POBN with $0.1 \mathrm{M}$ 4-oxo-TEMP, the ESR peak heights of 4oxo-TEMPO decreased, AMP dose-dependently (Fig. 7). At $2 \mathrm{mM}$ of AMP, the ESR peak heights of 4-oxo-TEMPO decreased to $27.3 \pm 0.6 \%$ of the standard reaction mixture of FMN without $\mathrm{Fe}^{2+}$ and 4-POBN with $0.1 \mathrm{M}$ 4-oxo-TEMP. On the other hand, the ESR peak height of the 4-POBN radical adduct also decreased with the increase of the concentration of AMP (Fig. 7). At $2 \mathrm{mM}$ of AMP, the ESR peak heights of 4-POBN radical adduct decreased to $51 \pm 6 \%$ of the standard reaction mixture of FMN.

The effects of the other nucleotides (GMP or CMP or TMP) on the ESR peak height of the 4-POBN radical adduct were investigated for the standard reaction mixtures of FMN with $2 \mathrm{mM}$ GMP (or CMP or TMP) (Table 2). On addition of $2 \mathrm{mM}$ GMP, the ESR peak heights of 4-POBN radical adduct decreased to $37 \pm 11 \%$ of the standard reaction mixture of FMN. The CMP and TMP showed no inhibitory effect.

HPLC-ESR analyses of the standard reaction mixture of FMN under the UVA irradiation. In order to determine the structure of the radicals formed in the standard reaction mixtures

Table 2. Effects of nucleotids on the ESR peak heights of the 4-POBN radical adducts

\begin{tabular}{cc}
\hline Compounds added & Relative ESR peak height (\%) \pm SD \\
\hline AMP & $51 \pm 6$ \\
GMP & $37 \pm 11$ \\
CMP & $110 \pm 2$ \\
TMP & $94 \pm 8$
\end{tabular}

The reaction was performed for the standard reaction mixture of FMN with $2 \mathrm{mM}$ AMP (or GMP or CMP or TMP). The data presented are mean \pm SD of three independent experiments. The relative ESR peak heights $(\%)$ are percentages in comparison with the ESR peak height of the standard reaction mixture of FMN.

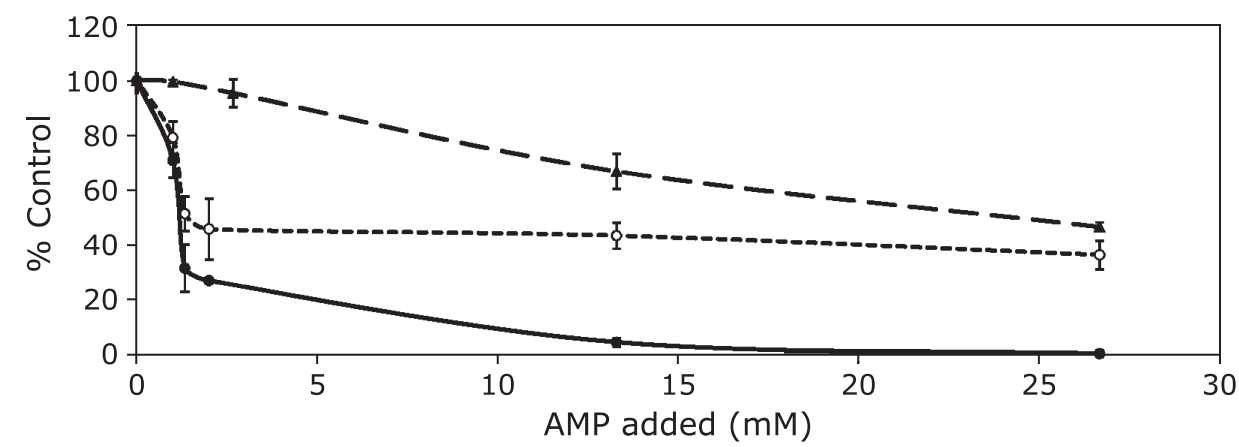

Fig. 7. The effect of AMP on the fluorescence spectra and the ESR peak height of the 4-POBN radical adduct and 4-oxo-TEMPO. The measurements of fluorescence spectra were done for the standard reaction mixtures of FMN without $\mathrm{Fe}^{2+}$ and 4-POBN with various concentration of AMP (----). The data presented are the percentages in comparison with the one of the standard reaction mixtures of FMN without Fe ${ }^{2+}, 4-\mathrm{POBN}$, and AMP. ESR measurements of 4-POBN radical adduct were performed for the standard reaction mixtures of FMN with various concentration of AMP (-----). ESR spectra of 4-oxo-TEMPO were observed for the standard reaction mixture without Fe ${ }^{2+}$ and 4-POBN with $0.1 \mathrm{M} 4-0 \times 0-\mathrm{TEMP}$ and various concentrations of AMP $(-)$. The data presented for the 4-oxo-TEMPO and the 4-POBN radical adducts are the percentages in comparison with the one of the standard reaction mixtures of FMN without AMP. 


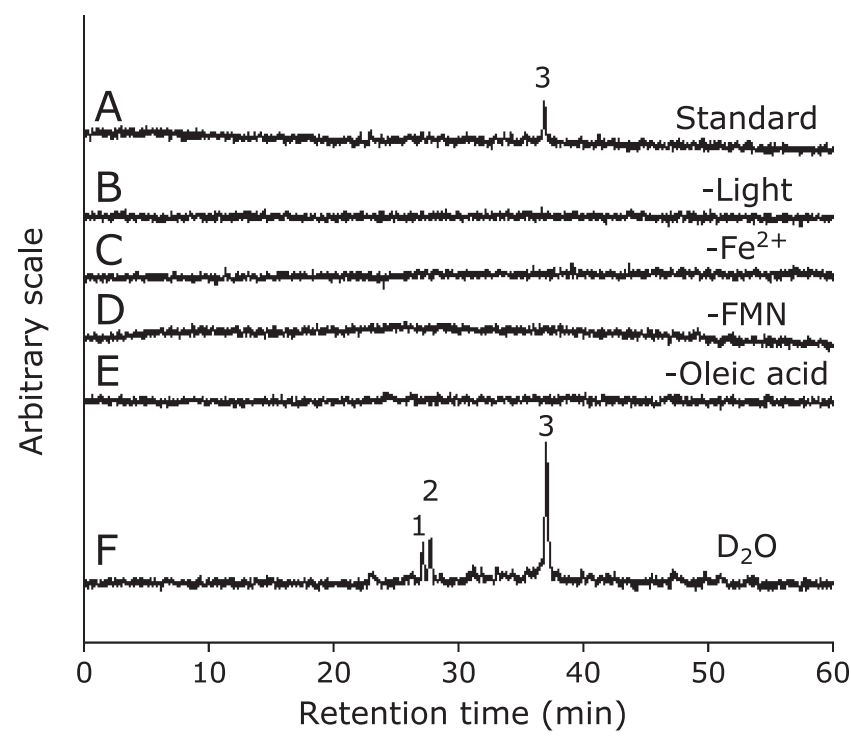

Fig. 8. HPLC-ESR analyses of the standard reaction mixtures of FMN. The total volume of the reaction mixture was $3.0 \mathrm{ml}$. The standard reaction mixture of $F M N(A), F M N$ without light (B), FMN without $\mathrm{Fe}^{2+}(\mathrm{C}), \mathrm{FMN}$ without FMN (D), FMN without oleic acid (E), and FMN in $\mathrm{D}_{2} \mathrm{O}(\mathrm{F})$.

of FMN under the UVA irradiation, the HPLC-ESR analyses were performed for the standard reaction mixtures of FMN. A prominent peak (peak 3) was observed on the HPLC-ESR elution profile of the standard reaction mixture of FMN under the UVA irradiation (Fig. 8A). The retention time of the peak 3 is $36.9 \mathrm{~min}$. The peak 3 was hardly observed for the standard reaction mixture of FMN without the UVA irradiation (or $\mathrm{Fe}^{2+}$ or $\mathrm{FMN}$ or oleic acid) (Fig. 8 B-E).

The standard reactions of FMN were performed in $\mathrm{D}_{2} \mathrm{O}$ (Fig. 8F). The HPLC-ESR analyses of the reaction mixtures of FMN in $\mathrm{D}_{2} \mathrm{O}$ showed that peak height of the peak 3 increased to $297 \pm 26 \%$ of the one in $\mathrm{H}_{2} \mathrm{O}$ (Fig. 8F). Thus, singlet oxygen seems to be involved in the formation of the radical of the peak 3 because $\mathrm{D}_{2} \mathrm{O}$ increases the lifetime of singlet oxygen. ${ }^{(3)}$ Two new peaks (peak 1 and peak 2) appeared at the retention times of 26.8 and $27.3 \mathrm{~min}$ in the standard reaction mixture of FMN in $\mathrm{D}_{2} \mathrm{O}$.

HPLC-ESR-MS analyses of the standard reaction mixture of FMN in $\mathrm{D}_{2} \mathrm{O}$. In order to determine the structure of peak 1 , peak 2 and peak 3 compounds observed on the HPLC-ESR elution profile of the standard reaction mixture of FMN in $\mathrm{D}_{2} \mathrm{O}$, the HPLC-ESR-MS analyses were performed. The HPLC-ESR-MS analysis of the peak 1 compound gave ions at $\mathrm{m} / \mathrm{z} 354$ (Fig. 9A). We tentatively assigned the ions at $\mathrm{m} / \mathrm{z} 354$ to the protonated molecules of $4-\mathrm{POBN} /{ }^{\circ} \mathrm{C}_{8} \mathrm{O}_{3} \mathrm{H}_{15}$ adduct. The HPLC-ESR-MS analysis of the peak 2 compound gave also ions at $\mathrm{m} / \mathrm{z} 354$ (Fig. 9B). The HPLC-ESR-MS analysis of the peak 3 compound gave ions at $\mathrm{m} / \mathrm{z} 251$ and $\mathrm{m} / \mathrm{z} 338$ (Fig. 9C). The ions at $\mathrm{m} / \mathrm{z} 338$ correspond to the protonated molecules of the 4-POBN/7carboxyheptyl radical adducts, $[\mathrm{M}+\mathrm{H}]^{+}$. The fragment ions at $\mathrm{m} / \mathrm{z}$ 251 correspond to the loss of $\left(\mathrm{CH}_{3}\right)_{3} \mathrm{C}(\mathrm{O}) \mathrm{N}$ from the protonated molecules.

HPLC-ESR and HPLC-ESR-MS analyses of the standard reaction mixtures of $F A D$ and folic acid in $D_{2} O$ under the UVA irradiation. To know which radicals formed in the standard reaction mixtures of FAD and folic acid under the UVA irradiation, the HPLC-ESR analyses were performed (Fig. 10) A peak (peak 3) appeared at the retention time of $37.0 \mathrm{~min}$ on the elution profile of the standard reaction mixtures of FAD in $\mathrm{D}_{2} \mathrm{O}$ (Fig. 10C). Based on the retention time, the peak 3 could be the 4-POBN/7-
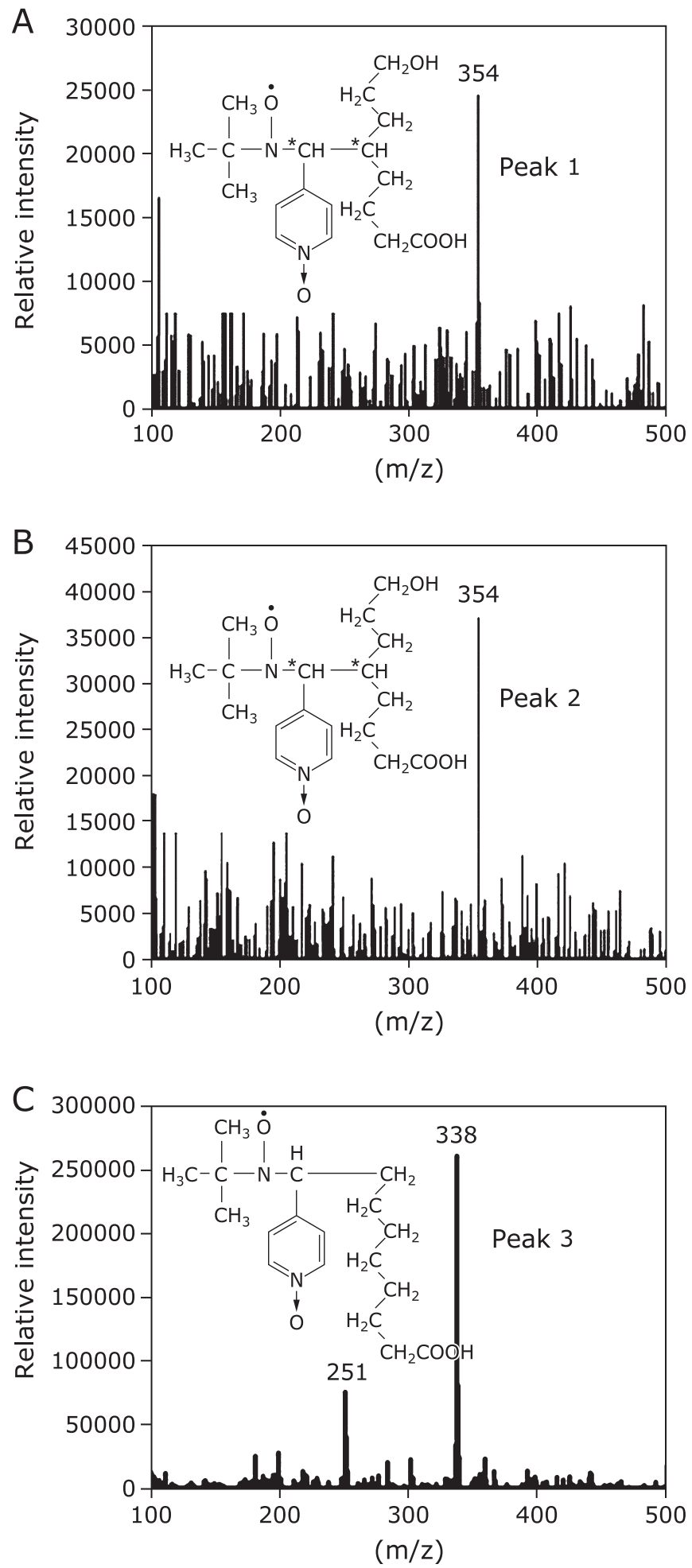

Fig. 9. HPLC-ESR-MS analyses of the peaks 1,2, and 3 observed in the standard reaction mixtures of FMN in $\mathrm{D}_{2} \mathrm{O}$. The total volume of the reaction mixture was $3.0 \mathrm{ml}$. (A) Peak 1. (B) Peak 2. (C) Peak 3. Chemical structures of 4-POBN/1-(3-carboxypropyl)-4-hydroxybutyl radical adducts and 4-POBN/7-carboxyheptyl radical adduct were inserted in (A), (B), and (C). Asymmetric carbon atoms of 4-POBN/1-(3-carboxypropyl)-4hydroxybutyl radical adducts are indicated by * marks. 
carboxyheptyl radical adducts. Three peaks (peak 1, peak 2 , and peak 3) appeared at the retention times of $27.1 \mathrm{~min}, 27.7 \mathrm{~min}$, and 36.9 min on the elution profile of the standard reaction mixtures of folic acid in $\mathrm{D}_{2} \mathrm{O}$ (Fig. 10D). The elution profile resembles the

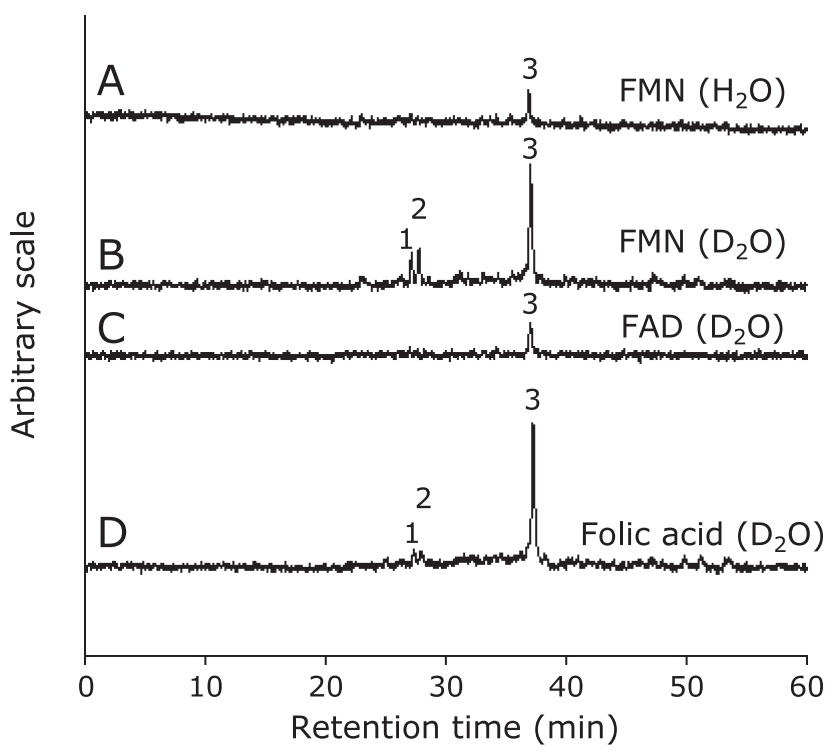

Fig. 10. HPLC-ESR analyses of the standard reaction mixtures of FMN $F A D$, and folic acid under the UVA irradiation. Total volume of the reaction mixture was $3 \mathrm{ml}$. The reactions were performed in $\mathrm{H}_{2} \mathrm{O}$ (or $\mathrm{D}_{2} \mathrm{O}$ ). HPLC-ESR analyses of the standard reaction mixtures of FMN in $\mathrm{H}_{2} \mathrm{O}(\mathrm{A}), \mathrm{FMN}$ in $\mathrm{D}_{2} \mathrm{O}(\mathrm{B}), \mathrm{FAD}$ in $\mathrm{D}_{2} \mathrm{O}(\mathrm{C})$, and folic acid in $\mathrm{D}_{2} \mathrm{O}(\mathrm{D})$. one of the HPLC-ESR analysis of FMN in $\mathrm{D}_{2} \mathrm{O}$. The HPLC-ESRMS analyses of the peak 1, peak 2, and peak 3 gave the ions at $\mathrm{m} / \mathrm{z} 354, \mathrm{~m} / \mathrm{z} 354$, and $\mathrm{m} / \mathrm{z} 338$, respectively (data not shown), suggesting that the same radical species form in the standard reaction mixture of folic acid as FMN.

\section{Discussion}

Our previous studies showed that 7-carboxyheptyl radicals form in the reaction mixture of riboflavin with oleic acid under UVA irradiation. $^{(24)}$ In this paper, we investigated the reactions of some endogenous photosensitizers (FMN or FAD or folic acid or NAD or NADP or PLP or urocanic acid) with oleic acid under UVA irradiation using ESR, HPLC-ESR, and HPLC-ESRMS. Prominent ESR signals $\left(\alpha^{\mathrm{N}}=1.58 \mathrm{mT}\right.$ and $\left.\alpha^{\mathrm{H}} \beta=0.26 \mathrm{mT}\right)$ were observed for the standard reaction mixture of FMN (or FAD or folic acid) (Fig. $1 \mathrm{~A}-\mathrm{D}$ ), suggesting that free radicals form in the standard reaction mixture of FMN (or FAD or folic acid). The HPLC-ESR and HPLC-ESR-MS analyses have shown that 7carboxyheptyl and ${ }^{\circ} \mathrm{C}_{8} \mathrm{O}_{3} \mathrm{H}_{15}$ radicals form in the standard reaction mixtures of FMN (or FAD or folic acid) under the UVA irradiation. When the reactions were performed in $\mathrm{D}_{2} \mathrm{O}$, the ESR and HPLC-ESR peak heights increased. Thus, singlet oxygen seems to be involved in the formation of the radicals because $\mathrm{D}_{2} \mathrm{O}$ increases the lifetime of singlet oxygen. ${ }^{(3)}$ Of the photosensitizers, NAD (or NADP or PLP or urocanic acid) did not induce the formation of the radicals. These may be due to their low quantum yields of the singlet oxygen generation. ${ }^{(5)}$ In spite of the low quantum yield of singlet oxygen generation, ${ }^{(27)}$ folic acid enhanced the formation of the radicals. These might have occurred through the photodegradation products of folic acid, 6-formylpterin and 6carboxypterin which show high quantum yields of the singlet oxygen generation. ${ }^{(28,29)}$

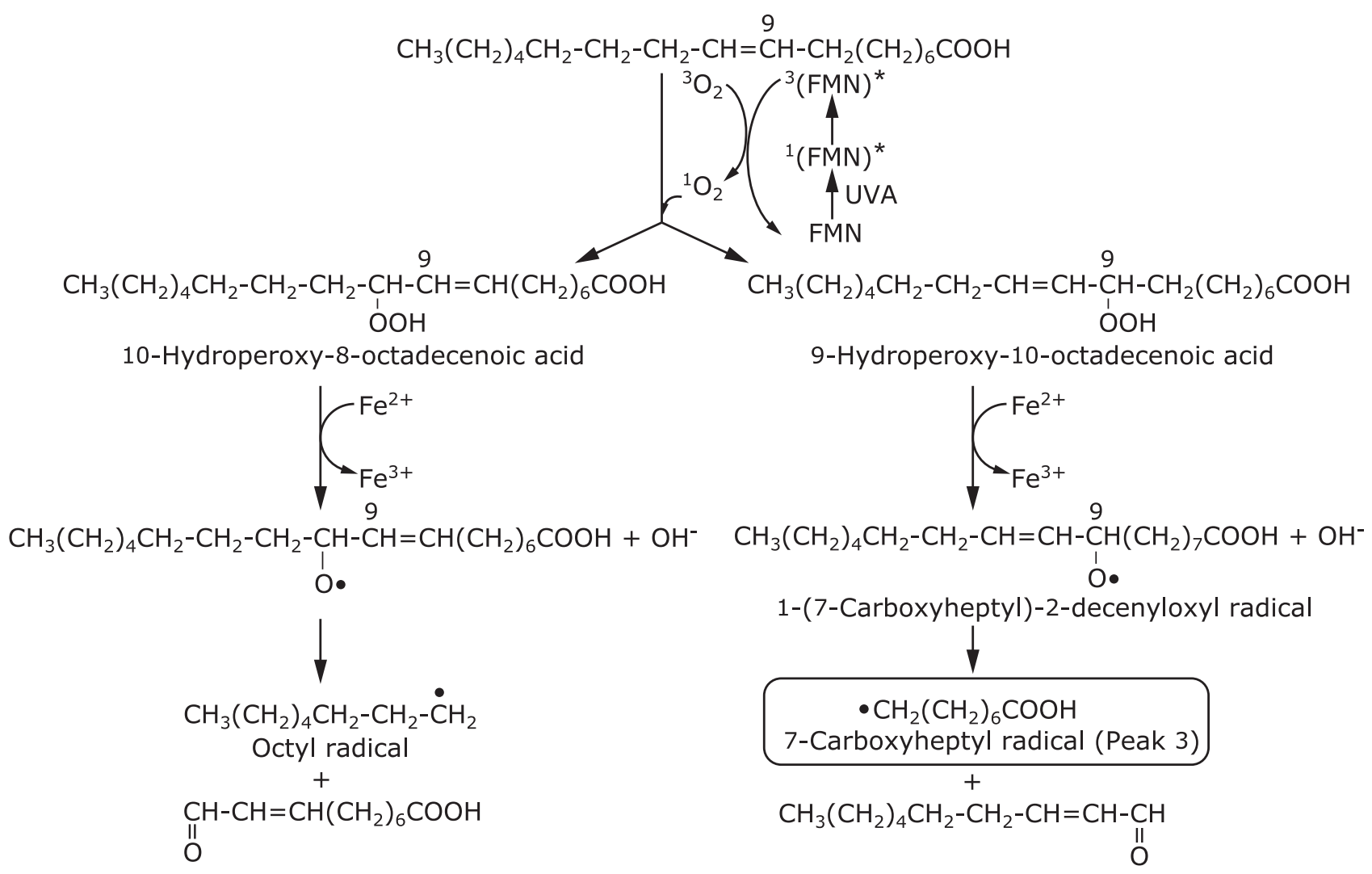

Fig. 11. A possible reaction path for the formation of the 7-carboxyheptyl radical (Peak 3) in the standard reaction mixture of FMN. 
A possible reaction path for the formation of the 7-carboxyheptyl radical is as follows (Fig. 11). Since irradiation of UVA is essential for the formation of the radicals in the standard reaction mixture of FMN (Fig. 2 and 3), the excited singlet state of FMN ${ }^{1}(\mathrm{FMN}) *$ seems to participate in the formation of the radicals. The excited singlet state sensitizer ${ }^{1}(\mathrm{FMN})^{*}$, which is produced under the UVA irradiation turns to the excited triplet state ${ }^{3}(\mathrm{FMN})^{*}$ through intersystem crossing. The excited triplet state ${ }^{3}(\mathrm{FMN})^{*}$ may reacts with triplet oxygen to form singlet oxygen (Eq. 1). ${ }^{(5,24)}$

${ }^{3}(\mathrm{FMN}) *+{ }^{3} \mathrm{O}_{2} \rightarrow \mathrm{FMN}+{ }^{1} \mathrm{O}_{2}$

The reaction of oleic acid with the singlet oxygen results in the formation of LOOH (9-hydroperoxy-10-octadecenoic acid or 10hydroperoxy-8-octadecenoic acid) through the singlet oxygen ene reaction. ${ }^{(30)}$ Since ESR signals were not observed in the standard reaction mixture of FMN without ferrous ions (Fig. 2), ferrous ions seem to participate in the formation of the radicals. The ferrous ions possibly catalyze decomposition of 9-hydroperoxy10 -octadecenoic acid. This reaction yields a radical intermediate LO [1-(7-carboxyheptyl)-2-decenyloxyl radical]. The $\beta$-scession of the $\mathrm{LO}^{*}$ could result in the generation of the 7-carboxyheptyl radical. The reaction of ferrous ion with 10-hydroperoxy-8octadecenoic acid could result in the formation of octyl radicals. We could not detect the radicals. Since 4-POBN used in this experiment is a relatively polar molecule, the octyl radicals which are located at the non-polar area may not be trapped by 4-POBN.

Molecular structures of the peak 1 and peak 2 compounds, which were observed in the HPLC-ESR elution profiles of the standard reaction mixtures of FMN (or folic acid), may be assigned as the two diastereoisomers of 4-POBN/1-(3-carboxypropyl)-4hydroxybutyl radical adducts (Fig. 9). A possible reaction path for the formation of the 1-(3-carboxypropyl)-4-hydroxybutyl radical is as follows (Fig. 12). The 8-hydroperoxyoctanoic acid could form through the reaction of 7-carboxyheptyl radicals, of which formation was described above (Fig. 11) with molecular oxygens. The ferrous ions possibly catalyze decomposition of the 8hydroperoxyoctanoic acid. This reaction yields a radical intermediate, 7-carboxyheptyloxyl radical. Isomerization of the 7carboxyheptyloxyl radicals by 1,5 -hydrogen shift via a low-strain six-membered ring transition state possibly results in the formation of 1-(3-carboxypropyl)-4-hydroxybutyl radicals. ${ }^{31,32)}$

To know why FMN works as a potent photosensitizer compared with FAD, effects of adenosine moiety on the formation of the radicals and singlet oxygen in addition to the fluorescence spectrum of FMN were examined. Formation of the radicals and singlet oxygen decreased with the increase of the AMP concentration in a similar manner. On addition of AMP to the reaction mixtures, peak height of the fluorescence spectra also decreased with the increase of the AMP concentration, but in a quite different manner as the ESR peak heights of the 4-POBN radical adducts and the 4-oxo-TEMPO, indicating that the quenching of the excited singlet ${ }^{1}(\mathrm{FMN})^{*}$ does not seem to affect directly the formation of the oleic acid-derived radicals and singlet oxygen. Since singlet oxygen participates in the formation of the oleic acid-derived radicals (Table 1), the AMP may scavenge singlet oxygen. At higher concentrations of AMP, ESR peak heights of the 4-POBN radical adduct remained unchanged. The chemical species other than singlet oxygen may participate in the formation of the 4-POBN radical adducts at the higher concentration of AMP.

When irradiation energy dependence of the ESR peak height was measured for FMN and FAD, quite different dependences of the ESR peak height were observed for FMN and FAD (Fig. 5). FAD reached a plateau $(1.0 \mathrm{~cm})$ at $1.95 \mathrm{~J} / \mathrm{cm}^{2}$, while FMN reached a plateau $(2.8 \mathrm{~cm})$ at $15.6 \mathrm{~J} / \mathrm{cm}^{2}$. The difference seems to be due to the adenosine moiety in FAD. The reaction between the adenosine moiety and the excited isoalloxazine ring may result in the<smiles>CCCCCCCC(=O)O</smiles>

Fig. 12. A possible reaction path for the formation of the 1-(3-carboxypropyl)-4-hydroxybutyl radical (peaks 1 and 2) in the standard reaction mixture of FMN.

degradation of FAD and the irradiation energy dependence of the ESR peak height.

\section{Abbreviations}

$\begin{array}{ll}\text { AMP } & \text { 2'-deoxyadenosine-5'-monophospate } \\ \text { CMP } & \text { 2'-deoxycytidine-5'-monophospate } \\ \text { DMPO } & \text { 5,5-dimethyl-1-pyrroline- } N \text {-oxide } \\ \text { ESR } & \text { electron spin resonance } \\ \text { FAD } & \text { flavin adenine dinucleotide } \\ \text { FMN } & \text { flavin mononucleotide } \\ \text { GMP } & \text { 2'-deoxyguanosine-5'-monophospate } \\ \text { HPLC-ESR } & \begin{array}{l}\text { high performance liquid chromatography- } \\ \text { electron spin resonance }\end{array} \\ \text { HPLC-ESR-MS } & \begin{array}{l}\text { high performance liquid chromatography- } \\ \text { electron spin resonance-mass spectrometry }\end{array} \\ \text { NAD } & \begin{array}{l}\beta \text {-nicotinamide adenine dinucleotide } \\ \text { NADP }\end{array} \\ \text { 4-oxo-TEMP } & \text { 4-oxo-2,2,6,6-tetramethylpiperidine } \\ \text { 4-oxo-TEMPO } & \text { 4-oxo-2,2,6,6-tetramethylpiperidine- } N \text {-oxyl }\end{array}$


PLP

4-POBN

ROS

TMP

UVA

pyridoxal-5'-phosphate

$\alpha$-(4-pyridyl-1-oxide)- $N$-tert-butylnitrone

reactive oxygen species

thymidine-5'-monophospate

ultraviolet light A

\section{Conflict of Interest}

No potential conflicts of interest were disclosed.

\section{References}

1 Yasui H, Sakurai H. Chemiluminescent detection and imaging of reactive oxygen species in live mouse skin exposed to UVA. Biochem Biophys Res Comm 2000; 269: 131-136.

2 Yasui H, Sakurai H. Age-dependent generation of reactive oxygen species in the skin of live hairless rats exposed to UVA light. Exp Dermatol 2003; 12: 655-661.

3 Schweitzer C, Schmidt R. Physical mechanisms of generation and deactivation of singlet oxygen. Chem Rev 2003; 103: 1685-1757.

4 Ragas X, Agut M, Nonell S. Singlet oxygen in Escherichia coli: new insights for antimicrobial photodynamic therapy. Free Radic Biol Med 2010; 49: 770776.

5 Baier J, Maisch T, Maier M, Engel E, Landthaler M, Bäumler W. Singlet oxygen generation by UVA light exposure of endogenous photosensitizers. Biophys J 2006; 91: 1452-1459.

6 Hanson KM, Simon JD. Epidermal trans-urocanic acid and the UV-Ainduced photoaging of the skin. Proc Natl Aca Sci USA 1998; 95: 1057610578.

7 Dzhagarov BM, Kruk NN, Konovalova NV, Solodunov AA, Stepuro II. Quantum yield of photosensitized formation of singlet oxygen by vitamins of the B6 group and their adducts with amino acids and proteins. J Appl Spectrosc 1994; 61: 505-508.

8 Tanaka M, Ohkubo K, Fukuzumi S. DNA cleavage by UVA irradiation of NADH with dioxygen via radical chain processes. J Phys Chem A 2006; 110: 11214-11218.

9 Yamazaki S, Ozawa N, Hiratsuka A, Watabe T. Photogeneration of $3 \beta-$ hydroxy-5 $\alpha$-cholest-6-ene-5-hydroperoxide in rat skin: evidence for occurrence of singlet oxygen in vivo. Free Radic Biol Med 1999; 27: 301-308.

10 Chacon JN, McLearie J, Sinclair RS. Singlet oxygen yields and radical contributions in the dye-sensitised photo-oxidation in methanol of esters of polyunsaturated fatty acids (oleic, linoleic, linolenic and arachidonic). Photochem Photobiol 1988; 47: 647-656.

11 Bando N, Hayashi H, Wakamatsu S, et al. Participation of singlet oxygen in ultraviolet-a-induced lipid peroxidation in mouse skin and its inhibition by dietary $\beta$-carotene: an ex vivo study. Free Radic Biol Med 2004; 37: 1854 1863.

12 Minami Y, Yokoyama N, Bando N, Kawai Y, Terao J. Occurrence of singlet oxygen oxygenation of oleic acid and linoleic acid in the skin of live mice. Free Radic Res 2008; 42: 197-204.

13 Watabe N, Ishida Y, Ochiai A, Tokuoka Y, Kawashima N. Oxidation decomposition of unsaturated fatty acids by singlet oxygen in phospholipid bilayer membranes. J Oleo Sci 2007; 56: 73-80.

14 Mazari A, Iwamoto S, Yamauchi R. Ultraviolet A-induced peroxidation of phosphatidylcholine in unilamellar liposomes. Biosci Biotechnol Biochem 2009; 73: 1212-1214.

15 Huvaere K, Cardoso DR, Homem-de-Mello P, Westermann S, Skibsted LH. Light-induced oxidation of unsaturated lipids as sensitized by flavins. J Phys Chem B 2010; 114: 5583-5593.

16 Taira J, Mimura K, Yoneya T, Hagi A, Murakami A, Makino K. Hydroxyl radical formation by UV-irradiated epidermal cells. J Biochem 1992; 111: 693-695.

17 Xia Q, Yin JJ, Cherng SH, et al. UVA photoirradiation of retinyl palmitateformation of singlet oxygen and superoxide, and their role in induction of lipid peroxidation. Toxicol Lett 2006; 163: 30-43.

18 Nishi J, Ogura R, Sugiyama M, Hidaka T, Kohno M. Involvement of active oxygen in lipid peroxide radical reaction of epidermal homogenate following ultraviolet light exposure. J Invest Dermatol 1991; 97: 115-119.

19 Ogura R, Sugiyama M, Nishi J, Haramaki N. Mechanism of lipid radical formation following exposure of epidermal homogenate to ultraviolet light. $J$ Invest Dermatol 1991; 97: 1044-1047.

20 Jurkiewicz BA, Buettner GR. Ultraviolet light-induced free radical formation in skin: an electron paramagnetic resonance study. Photochem Photobiol 1994; 59: 1-4.

21 Haywood R, Rogge F, Lee M. Protein, lipid, and DNA radicals to measure skin UVA damage and modulation by melanin. Free Radic Biol Med 2008; 44: $990-1000$.

22 Haywood R, Andrady C, Kassouf N, Sheppard N. Intensity-dependent direct solar radiation- and UVA-induced radical damage to human skin and DNA, lipids and proteins. Photochem Photobiol 2011; 87: 117-130.

23 Iwahashi H. High performance liquid chromatography/electron spin resonance/mass spectrometry analyses of lipid-derived radicals. In: Armstrong D, ed. Methods in Molecular Biology. Advanced Protocols in Oxidative Stress New York: Humana Press, 2008; 477: 65-73.

24 Mori H, Iwahashi H. Formation of 7-carboxyheptyl radical induced by singlet oxygen in the reaction mixture of oleic acid, riboflavin and ferrous ion under the UVA irradiation. J Clin Biochem Nutr 2011; 49: 141-146.

25 Lion Y, Delmelle M, Van de Vorst A. New method of detecting singlet oxygen production. Nature $1976 ; 263$ : 442-443.

26 Nakamura K, Ishiyama K, Ikai H, et al. Reevaluation of analytical methods for photogenerated singlet oxygen. J Clin Biochem Nutr 2011; 49: 87-95.

27 Tomas AH, Lorente C, Capparelli AL, Martinez CG, Braun AM, Oliveros E. Singlet oxygen $\left({ }^{1} \Delta \mathrm{g}\right)$ production by pterin derivatives in aqueous solutions. Photochem Photobiol Sci 2003; 2: 245-250.

28 Akhtar MJ, Khan MA, Ahmad I. Photodegradation of folic acid in aqueous solution. J Pharm Biomed Anal 1999; 19: 269-275.

29 Dantola ML, Denofrio MP, Zurbano B, et al. Mechanism of photooxidation of folic acid sensitized by unconjugated pterins. Photochem Photobiol Sci 2010; 9: 1604-1612.

30 Stephenson LM. The mechanism of the singlet oxygen ene reaction. Tetrahedron Lett 1980; 21: 1005-1008.

31 Carter WPL, Darnall KR, Lloyd AC, Winer AM, Pitts JN Jr. Evidence for alkoxy radical isomerization in photooxidations of $\mathrm{C} 4-\mathrm{C} 6$ alkanes under simulated atmospheric conditions. Chem Phys Lett 1976; 42: 22-27.

32 Niki H, Maker PD, Savage CM, Breitenbach LP. An FT IR study of the isomerization and $\mathrm{O}_{2}$ reaction of n-butoxy radicals. $J$ Phys Chem 1981; 85: 2698-2700. 